\title{
GMR
}

\section{Tumor-suppressive role of Kruppel-like factor 4 (KLF-4) in colorectal cancer}

\author{
D.H. Xiu ${ }^{1 *}$, Y. Chen ${ }^{2 *}$, L. Liu ${ }^{1}$, H.S. Yang ${ }^{1}$ and G.F. Liu ${ }^{1}$ \\ ${ }^{1}$ Department of Radiology, China-Japan Union Hospital of Jilin University, \\ Changchun, China \\ ${ }^{2}$ Department of Endocrinology, Second Hospital of Jilin University, \\ Changchun, China \\ *These authors contributed equally to this study. \\ Corresponding author: G.F. Liu \\ E-mail: jlfsliuguifeng@163.com \\ Genet. Mol. Res. 16 (1): gmr16019272 \\ Received September 13, 2016 \\ Accepted February 8, 2017 \\ Published February 16, 2017 \\ DOI http://dx.doi.org/10.4238/gmr16019272
}

Copyright (C) 2017 The Authors. This is an open-access article distributed under the terms of the Creative Commons Attribution ShareAlike (CC BY-SA) 4.0 License.

\begin{abstract}
Kruppel-like factors (KLFs) are a group of transcriptional regulators that have recently been identified to exhibit tumorsuppressive function against various gastrointestinal cancers. The present study aims to investigate the expression patterns and prognostic value of KLF-4 in colorectal cancers (CRCs). KLF-4 levels in CRC tissues were examined via immunohistochemistry analysis, real-time quantitative polymerase chain reaction, and western blotting. The chisquare test was performed to evaluate the correlation between KLF-4 expression and the clinicopathological characteristics. Kaplan-Meier analysis was performed to assess the prognostic value of KLF-4 in CRC patients. In addition, we evaluated the effect of KLF-4 knockdown on the proliferation of CRC HT-29 cells. Our results showed significant downregulation of KLF-4 in 31 CRC samples, collected from CRC patients showing more malignant characteristics such as lymphatic metastasis, low tumor cell differentiation, and tumor recurrence. CRC patients in the low KLF-4 group were found to have reduced overall
\end{abstract}

Genetics and Molecular Research 16 (1): gmr16019272 
survival and decreased disease-free survival time. Moreover, HT-29 cells transfected with siRNA-KLF-4 showed increased proliferation compared to those transfected with control siRNA. In summary, lower KLF-4 expression was correlated with malignant CRC status and poor prognosis in CRC patients. Moreover, KLF-4 suppression promoted the proliferation of CRC cells in vitro. These results provide novel insights into the tumor suppressive role of KLF-4 in CRC.

Key words: Kruppel-like factor 4; Tumor suppression; Prognosis; Colorectal cancer

\section{INTRODUCTION}

Colorectal cancer (CRC) is the third most commonly occurring cancer and the fourth most common cause of cancer mortality globally (Balog et al., 2012; Abbruzzese et al., 2015; Weng et al., 2015). Multiple oncogenic markers have been identified in CRCs, namely, endothelial cell-specific molecule-1 (Jiang et al., 2015), transcription termination factor 1 (Ueda et al., 2015), spindle assembly abnormal protein 6 homolog (Shinmura et al., 2015), and baculoviral IAP repeat containing 6, which have been associated with poor prognosis in CRC patients. Increased levels of preoperative chemokine (C-C motif) ligand 2 (CCL-2) (Szczepanik et al., 2015), serpin peptidase inhibitor clade A member (Kwon et al., 2015), chemokine receptor 4 (Li et al., 2015a), or high mobility group box 1 (Zhang et al., 2015) in CRC patients have also been demonstrated to stimulate the proliferation of tumor cells and are correlated with the poor prognosis in CRC patients. On the other hand, several biomarkers were indicated to exhibit tumor suppressive function in CRC. Expression of cytokeratin 20 (Li et al., 2015b) and non-metastatic protein 23 (Fu and $\mathrm{Chu}, 2015$ ) have been correlated with higher survival rates. Kruppel-like factors (KLFs) are transcriptional regulators that are widely expressed in human tissues and exert diverse functions in cellular processes, such as proliferation, inflammation, differentiation, and migration (McConnell and Yang, 2010). KLF-4 is highly expressed in adult guts (Zheng et al., 2009) and act by regulating intestinal differentiation (Li et al., 2011). Recently, the tumor suppressive role of KLF-4 has been identified in various cancers, including gastric cancer (Zhang et al., 2012), prostate cancer (Shin et al., 2014), esophageal cancer (Zhang et al., 2009), and lung cancer (Zhou et al., 2010). KLF-4 acts by deregulating the malignant transformation and cellular hyperproliferation. Forced induction of KLF-4 expression has been shown to inhibit the differentiation in esophageal cells (Zheng et al., 2009). Moreover, restoration of KLF-4 expression can antagonize development and progression of intestinal tumors (Ghaleb et al., 2007). Consistently, a recent study clearly demonstrated that KLF-4 expression is a prognostic predictor for colon cancer (Patel et al., 2010; Lee et al., 2014), and reduced expression of KLF-4 promotes intestinal tumorigenic progression (Ghaleb et al., 2007). However, the mechanisms by which KLF-4 suppresses CRC tumorigenesis remains unclear. Therefore, we speculate that KLF-4 might pose tumor suppression in CRC. In this study, we examined KLF-4 expression in CRC samples, associated the KLF-4 expression with the prognosis of CRC patients, and evaluated the effects of KLF-4 insufficiency on the proliferation of CRC cells by knocking down KLF-4 expression in CRC cells. Our results demonstrated that reduced KLF-4 expression in human CRC tissues was associated with growth promotion of CRC cells.

Genetics and Molecular Research 16 (1): gmr16019272 


\section{MATERIAL AND METHODS}

\section{CRC samples, cell cultures, and reagents}

In total, $68 \mathrm{CRC}$ specimens were obtained from CRC patients subjected to radical resection and who received 5-FU-based chemotherapy after surgery, with complete clinicopathological data recorded for each specimen. Patient history was obtained, and each patient was subjected to physical surveillance monthly for the first year, every two months for 1-2 years, every four months for 2-3 years, every 6 months for up to 5 years, and finally annually. Detailed clinicopathological characteristics, including gender, age, location, $\mathrm{T}$ stage, $\mathrm{N}$ stage, differentiation, vessel invasion, and tumor recurrence, are listed in Table 1. CRC samples for use in scientific research were collected with permission from the donors. The present study was approved by the institutional ethics committee of our hospital. CRC HT-29 cells were purchased from American Type Culture Collection (ATCC) (Rockville, MD, USA) and cultured in the McCoy's 5a medium (GIBCO, Rockville, MD, USA) containing 10\% fetal bovine serum (Invitrogen, Carlsbad, CA, USA). HT-29 cells were cultured at $37^{\circ} \mathrm{C}$ with $5 \% \mathrm{CO}_{2}$. To silence KLF-4 expression, HT-29 cells were transfected with 25 or $50 \mathrm{nM} \mathrm{KLF-4-specific} \mathrm{siRNA} \mathrm{and} \mathrm{control} \mathrm{siRNA}$ (Ambion, Austin, TX, USA) using Lipofectamine RNAiMax (Invitrogen) for $6 \mathrm{~h}$. Next, culture media were replaced with fresh McCoy's 5a medium supplemented with $2 \%$ FBS, and cells were assayed for KLF-4 knockdown efficiency following another round of siRNA transfection for $24 \mathrm{~h}$ or were subjected to other treatments.

Table 1. Immunohistochemical staining results showing the correlation between KLF expression and clinicopathological characteristics.

\begin{tabular}{|c|c|c|c|c|c|}
\hline \multirow[t]{2}{*}{ Characteristics } & \multicolumn{2}{|c|}{ Low KLF } & \multicolumn{2}{|c|}{ High KLF } & \multirow[t]{2}{*}{ P value } \\
\hline & $\mathrm{N}$ & $\%$ & $\mathrm{~N}$ & $\%$ & \\
\hline Gender & & & & & 0.87786 \\
\hline Male & 19 & 61.29 & 22 & 59.46 & \\
\hline Female & 12 & 38.71 & 15 & 40.54 & \\
\hline Age (years) & & & & & 0.87394 \\
\hline$\leq 65$ & 14 & 45.16 & 16 & 43.24 & \\
\hline$>65$ & 17 & 54.84 & 21 & 56.76 & \\
\hline Location & & & & & 0.7401 \\
\hline Colon & 18 & 58.06 & 20 & 54.05 & \\
\hline Rectum & 13 & 41.94 & 17 & 45.95 & \\
\hline T stage & & & & & 0.91695 \\
\hline $\mathrm{T} 1+\mathrm{T} 2$ & 1 & 3.13 & 1 & 2.70 & \\
\hline $\mathrm{T} 3+\mathrm{T} 4$ & 31 & 96.88 & 36 & 97.30 & \\
\hline $\mathrm{N}$ stage & & & & & $0.02521 *$ \\
\hline$\leq \mathrm{N} 1$ & 10 & 32.26 & 22 & 59.46 & \\
\hline$>\mathrm{N} 1$ & 21 & 67.74 & 15 & 40.54 & \\
\hline Differentiation & 31 & & & & $0.03399 *$ \\
\hline High & 13 & 41.94 & 25 & 67.57 & \\
\hline Low & 18 & 58.06 & 12 & 32.43 & \\
\hline Vessel invasion & & & & & 0.94104 \\
\hline Positive & 27 & 87.10 & 32 & 86.49 & \\
\hline Negative & 4 & 12.90 & 5 & 13.51 & \\
\hline Tumor recurrence & & & & & $0.00207 * *$ \\
\hline Positive & 18 & 58.06 & 8 & 21.62 & \\
\hline Negative & 13 & 41.94 & 29 & 78.38 & \\
\hline
\end{tabular}

$* \mathrm{P}<0.05, * * \mathrm{P}<0.01$.

Genetics and Molecular Research 16 (1): gmr16019272 


\section{Immunohistochemical staining for KLF-4}

Immunohistochemical staining was performed to examine the KLF-4 expression. Specimen slides were immersed in citrate buffer $(\mathrm{pH} 6.0)$ for $10 \mathrm{~min}$ to retrieve the antigen and incubated for $2 \mathrm{~h}$ at room temperature with primary antibody against KLF-4 (1:100, Abcam, Cambridge, UK). Samples were then incubated with horseradish peroxidase-conjugated secondary antibodies (Sinobio, Beijing, China) for $30 \mathrm{~min}$ at room temperature. Specific binding to the KLF-4 antigen was counterstained with Mayer's hematoxylin. The intensity and degree of staining was independently evaluated by two pathologists in a blinded manner. The staining intensity scoring was as follows: 0 (no staining), 1 (mild staining), 2 (moderate staining), and 3 (intense staining). The staining area was scored as $0(0 \%), 1(1-25 \%), 2$ $(26-50 \%), 3(51-75 \%)$, or $4(76-100 \%)$ based on the percentage of positively stained cells. Specimens with moderate or intense staining and with staining area greater than $1 \%$ were classified under the high KLF-4 group.

\section{Whole-body magnetic resonance imaging}

For metastasis screening, magnetic resonance imaging (MRI) was performed using a 1.5-Tesla (T) imaging system (Magnetom Sonata ${ }^{\mathrm{TM}}$, Siemens Healthineers, Erlangen, Germany) with an amplitude of $40 \mathrm{mT} / \mathrm{m}$ and slew rate of $200 \mathrm{mT} \cdot \mathrm{m}^{-1} \cdot \mathrm{ms}^{-1}$. The MRI scan was performed from head to feet. Patients were examined on a fully MR-compatible rolling table platform (BodySURF ${ }^{\mathrm{TM}}$, MR-Innovation, Essen, Germany), which was mounted on the original table of the MR system and placed on roller bearings for manual movement in the $\mathrm{Z}$ direction. T2WI sagittal and axial scans were performed for each patient to identify the primary CRC and detect vessel invasion and lymph node metastasis.

\section{RNA extraction and real-time quantitative polymerase chain reaction assay}

mRNA was extracted from HT-29 cells using Trizol reagent (Life Technologies, Grand Island, NY, USA) and added with RNasin ${ }^{\circledR}$ Plus RNase Inhibitor (Promega, Madison, WI, USA). For real-time quantitative polymerase chain reaction (RT-qPCR) analysis, SYBR green RT-qPCR kit (Takara, Tokyo, Japan) was utilized to quantify the relative KLF-4 mRNA levels using $\beta$-actin as internal control. $\Delta \Delta^{\mathrm{Ct}}$ method was used for relative quantification (Livak and Schmittgen, 2001).

\section{Western blot analysis of KLF-4 expression}

CRC samples or HT-29 cells were homogenized and lysed with Cell Lysis Buffer (Cell Signaling Technology Inc., Danvers, MA, USA) supplemented with Protease Inhibitor Cocktail (Abcam). The same amount of each protein sample was separated by $8-12 \%$ sodium dodecyl sulfate-polyacrylamide gel electrophoresis and transferred to a polyvinylidene fluoride membrane (Millipore, Bedford, MA, USA). KLF-4 and $\beta$-actin were assayed by immunoblotting analysis with rabbit polyclonal antibody against human KLF-4 (1:300; Abcam) or $\beta$-actin (1:100; Abcam). Goat anti-rabbit IgG conjugated to horseradish peroxidase (Jackson ImmunoResearch, West Grove, PA, USA) were used for detection using ECL detection systems (Amersham Pharmacia Biotech, Amersham, UK). KLF-4 levels were presented relative to $\beta$-actin levels.

Genetics and Molecular Research 16 (1): gmr16019272 


\section{Statistical analysis}

Statistically significant differences in KLF-4 expression in mRNA or protein level were examined using the Student $t$-test (unpaired). Two-tailed chi-square test was performed to determine the significant differences in various clinicopathological characteristics between the high and low KLF-4 groups. The log-rank test was performed to generate the survival curves. A P value less than 0.05 was considered statistically significant. Statistical analyses were carried out using the GraphPad Prism 5 statistical software package (GraphPad Software, La Jolla, CA, USA).

\section{RESULTS}

\section{Reduced KLF-4 expression in CRC tissues is associated with tumor malignancy}

To investigate the KLF-4 expression and its regulatory role in CRC, we examined KLF-4 expression in $68 \mathrm{CRC}$ samples comprising 38 colon cancer tissues and 30 rectal cancer tissues. First, immunohistochemistry results demonstrated that KLF-4 was differentially expressed in CRC tissues. We divided the 68 specimens into high KLF-4 $(\mathrm{N}=37)$ (Figure $1 \mathrm{~A})$ and low KLF-4 $(\mathrm{N}=31)$ (Figure 1B) groups based on staining intensity and staining area. Next, we validated both KLF-4 mRNA and protein expression levels via RT-qPCR and western blotting, respectively. As indicated in Figure 1C, KLF-4 mRNA levels were also markedly higher in the high KLF-4 group $(\mathrm{P}<0.0001)$. These results were consistent with western blotting results showing differences in KLF-4 levels in the two groups of CRC specimens (Figure 1D and 1E).

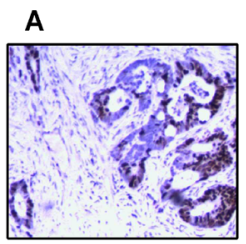

C

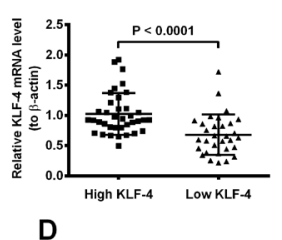

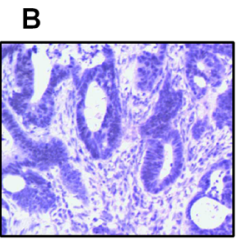

E

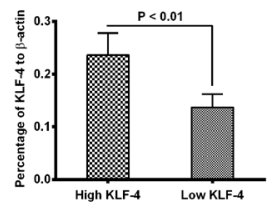

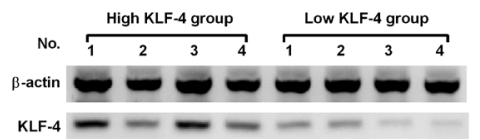

Figure 1. Representative immunohistochemical staining images showing KLF-4 expression in human colorectal cancer tissues. A. and B. Representative immunohistochemical staining results showing KLF-4 expression in colorectal cancers tissues $(\mathrm{N}=10)$. C. Representative mRNA levels of KLF-4 in the high LKF-4 $(\mathrm{N}=37)$ and the low LKF-4 (N=31) groups. D. and E. Western blotting assay of (D) and relative protein levels (E) of LKF-4 in the high LKF-4 $(\mathrm{N}=25)$ and the low LKF-4 $(\mathrm{N}=23)$ groups.

Genetics and Molecular Research 16 (1): gmr16019272 
To correlate KLF-4 expression with the clinicopathological characteristics of the CRC patients, we then analyzed the heterogeneity in gender, age, the location, stages ( $\mathrm{T}$ or $\mathrm{N}$ stage), differentiation invasion, and recurrence of CRC using the chi-square test. As indicated in Table 1, KLF-4 expression showed no significant correlation with gender, age, tumor location, T stage, or vessel invasion in CRC patients $(\mathrm{P}=0.87786,0.87394,0.7401,0.91695$, and 0.94104, respectively). However, lower KLF-4 expression was significantly associated with lymphatic metastasis, low tumor cell differentiation, and tumor recurrence $(\mathrm{P}=0.02521,0.03399$, and 0.00207 , respectively). Therefore, reduced KLF-4 expressionis associated withCRCmalignancy. To validate the association between KLF-4 downregulation and CRC malignancy, the number of metastatic lymph nodes based on MRI results of each CRC patient was counted and correlated with KLF-4 expression. Representative images of primary tumor and metastatic lymph nodes are shown in Figure 2. No significant difference in the primary tumor was found between the high KLF-4 group (Figure 2A) and the low KLF-4 group (Figure 2B) based on the T2WI sagittal scan. However, T2WI axial scans showed that samples in the low KLF-4 group had more metastatic lymph nodes compared to those in the high KLF-4 group (Figure 2D), and the differences between the two groups were found to be statistically significant (Figure 2E). Therefore, KLF-4 downregulation is associated with lymphatic CRC metastasis.
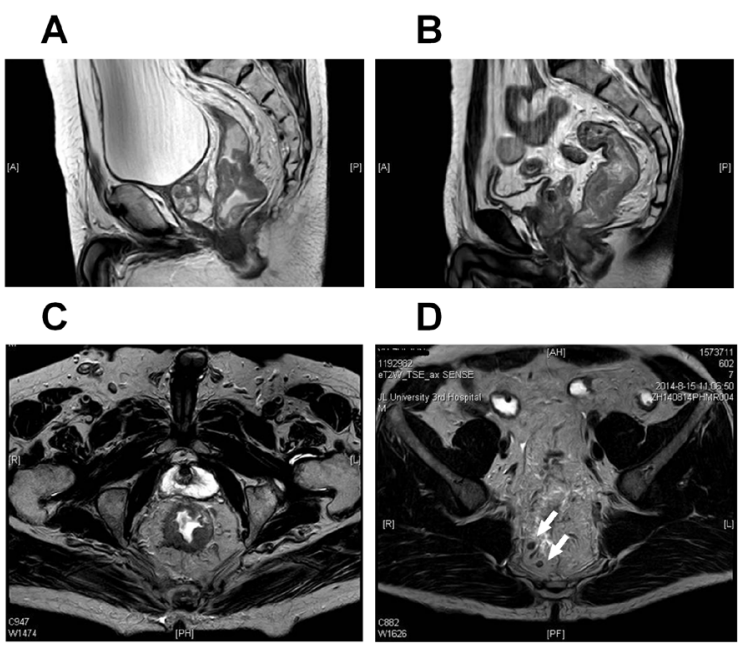

E

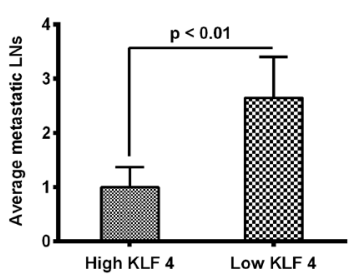

Figure 2. MRI results showing primary colorectal cancer and the lymphatic metastasis of CRCs. A. and B. T2WI sagittal scans for the primary tumor and its metastatic lymph nodes in patients from the high KLF-4 (A) and low KLF-4 groups (B). C. and D. T2WI axial scans for the primary tumor and metastatic lymph nodes in patients from the high KLF-4 (C) and the low KLF-4 groups (D). The primary tumor is labeled with a square marker, and metastatic lymph nodes are indicated by white arrows. E. Counts of metastatic lymph nodes. 


\section{KLF-4 downregulation is associated with poor prognosis in human CRC patients}

Kaplan-Meier analysis was performed to calculate the overall survival and disease-free survival of the two groups of CRC patients. Figure 3A shows that CRC patients with lower KLF-4 expression in cancer tissues had significantly reduced diseasefree survival time (DFS) than that for patients showing higher KLF-4 expression ( $\mathrm{P}=$ 0.0465 ). In addition, the overall survival (OS) of patients in the low KLF-4 group was also significantly lower than those in the high KLF-4 expression group $(\mathrm{P}=0.0126$, Figure 3B). Thus, our results suggest that KLF-4 is of clinical significance in the prognosis of CRC patients.
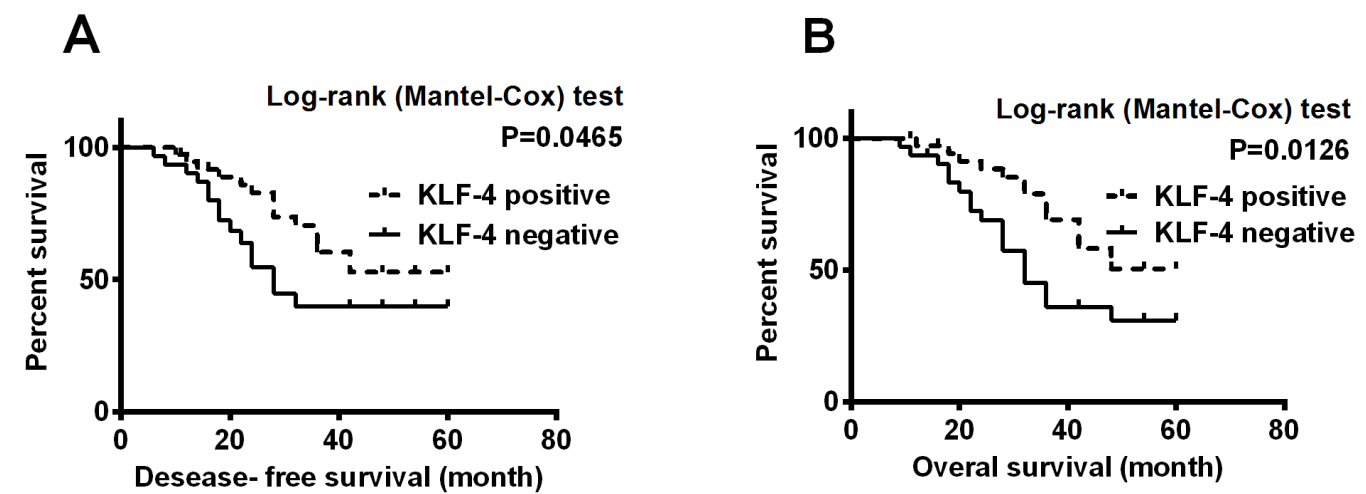

Figure 3. Log rank test analysis of survival for the $68 \mathrm{CRC}$ patients. A. Disease-free survival of patients in the high LKF-4 $(\mathrm{N}=37)$ and low LKF-4 $(\mathrm{N}=31)$ groups $(\mathrm{P}<0.05)$. B. Overall survival of patients in the high LKF-4 $(\mathrm{N}=$ $37)$ and the low LKF-4 $(\mathrm{N}=31)$ groups $(\mathrm{P}<0.05)$.

\section{RNAi-mediated KLF-4 knockdown promotes HT-29 cell proliferation}

To further investigate the mechanisms underlying the association between reduced KLF-4 expression and CRC malignancy, we knocked out KLF-4 expression in CRC HT-29 cells and measured cell growth. Figure 4A shows a significant reduction in KLF-4 mRNA expression in HT-29 cells transfected with KLF-4-specific siRNAs (siRNA-KLF-4) $(\mathrm{P}<0.01$ or $\mathrm{P}<0.001$ ). Western blotting results also showed marked abrogation of KLF-4 protein levels $(\mathrm{P}<0.01$ or $\mathrm{P}<0.001$, Figure 4B). Moreover, KLF-4 knockout significantly promoted the growth of HT-29 cells. siRNA-KLF-4-transfected HT-29 cells showed greater proliferation than that by cells transfected with siRNA-Con $(\mathrm{P}<0.05$ or $\mathrm{P}<0.01$, Figure $4 \mathrm{C})$. The increase in cell growth following KLF-4 knockdown was validated in HT-29 cells via the colony forming assay (Figure 4D). Both the colony number (Figure 4E) and the colony size (Figure 4F) were significantly higher in the siRNA-KLF-4 group than in the siRNA-Con group (colony number: $92.7 \pm 9.8$ vs $62.0 \pm 6.4, \mathrm{P}<0.05$; colony size: $0.273 \pm 0.026$ vs $0.160 \pm 0.021, \mathrm{P}<0.05$ ). Thus, growth promotion in CRC cells as a result of KLF-4 inhibition was reconfirmed via the loss-of-function strategy. 
A

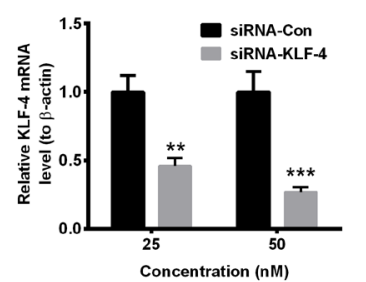

C
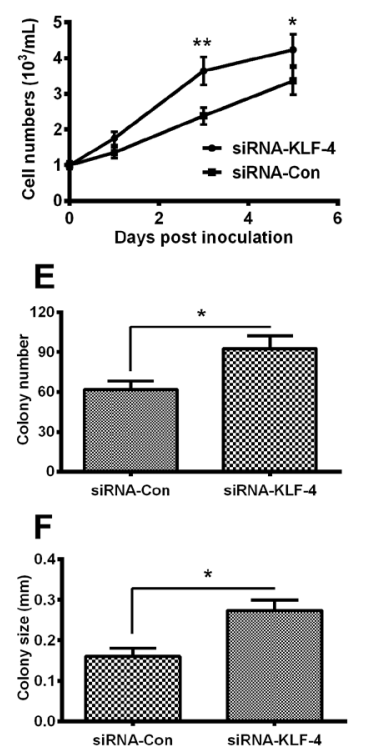

B

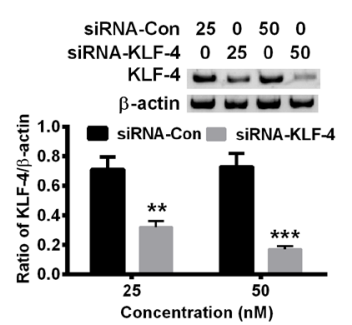

D

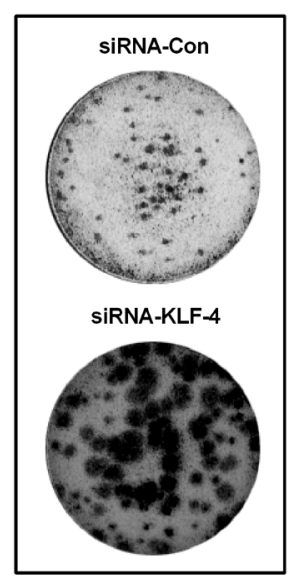

Figure 4. Transfection with KLF-4-specific siRNA promotes the proliferation of CRC cells in vitro. A. siRNAKLF-4 transfection for $24 \mathrm{~h}$ significantly suppressed the KLF-4 mRNA levels in HT-29 cells when compared to the siRNA control. KLF-4 mRNA expression was examined by quantitative real-time RT-PCR using $\beta$-actin as internal control. B. Western blotting to determine KLF-4 protein levels in HT-29 cells at $24 \mathrm{~h}$ post-transfection with KLF-4-specific siRNAs or siRNA control. C. Growth curve of HT-29 cells transfected with $50 \mathrm{nM}$ siRNA-KLF-4 or siRNA-Con. Cell numbers were counted at 1, 3, or 5 days post-inoculation. D. Colony-forming assay of HT-29 cells transfected with $50 \mathrm{nM}$ siRNA-KLF-4 or siRNA-Con. E. and F. Colony number or size of the siRNA-KLF-4or siRNA-Con-transfected HT-29 cells. Experiments were independently performed in triplicates. Results were considered statistically significant at $* \mathrm{P}<0.05, * * \mathrm{P}<0.01$, or $* * * \mathrm{P}<0.001$.

\section{DISCUSSION}

In the present study, we recognized the heterogeneity of KLF-4 expression in colorectal cancer (CRC) specimens. Immunohistochemistry analysis, real-time PCR, and western blotting results consistently confirmed reduced KLF-4 expression in 31 CRC samples. KLF4 reduction was shown to be associated with malignancy characteristics, such as lymphatic metastasis, low tumor cell differentiation, and tumor recurrence. Moreover, magnetic resonance imaging was performed to validate that CRC patients with lymph node metastasis had lower KLF-4 expression. In addition, Kaplan-Meier analysis of the overall survival and disease-free survival of the two groups of CRC patients demonstrated that patients with lower 
KLF-4 expression in their cancer tissues had reduced disease-free survival time and decreased overall survival, thereby suggesting the clinical significance of KLF-4 downregulation as an indicator of poor prognosis in CRC patients. However, only 68 samples were examined for KLF4 expression at the mRNA level. For analysis of KLF-4 protein levels, 48 samples were analyzed by western blotting, and 10 samples were analyzed by immunohistochemical analysis. More clinical samples must be analyzed to support our conclusion. It is not clear whether the tumor suppressive role of KLF-4 depends on other oncogenic or tumor suppressive biomarkers in CRC. Research into the mechanisms underlying the tumor suppressive function of KLF-4 is still ongoing. Accumulating evidence has indicated that KLF-4 sensitizes against oxidative DNA damage, mediates genetic stability, regulates cell cycle progression, and inhibits cancer cell proliferation. Oxidative DNA damage causes premature senescence in KLF-4-deficient mouse embryonic fibroblasts (Liu et al., 2015), and KLF-4 was demonstrated to regulate genetic stability in mouse embryonic fibroblasts (El-Karim et al., 2013). Attenuation of KLF-4 expression promotes carcinogenesis by inducing G1/S phase arrest in clear cell renal cell carcinoma (Song et al., 2013). KLF-4 blocks tumor cell proliferation and promotes drug resistance in multiple myeloma (Schoenhals et al., 2013). In the present study, we investigated the regulatory role of KLF-4 in the proliferation of CRC HT-29 cells by abrogating KLF-4 expression. Our results demonstrated that the RNAi-mediated KLF-4 knockdown promoted the proliferation of HT-29 cells based on growth curve assay and colony forming assay. A higher level of cell growth in HT29 cells was observed in the KLF-4 knockdown group. Thus, we confirmed that KLF-4 exerts its tumor suppressive role by regulating the growth of CRC cells. Our study implies that reduced KLF-4 expression is associated with increased cell proliferation. Thus, KLF-4 expression levels could serve as important biomarkers for CRC malignancy.

\section{CONCLUSIONS}

In summary, our study confirmed the significant reduction of KLF-4 expression in CRC tissues. KLF-4 inhibition was correlated with the malignant status of CRC and poor prognosis in CRC patients. Moreover, KLF-4 suppression promoted the proliferation of CRC cells in vitro. These results provide new insights into the tumor suppressive role of KLF-4 in CRC.

\section{Conflicts of interest}

The authors declare no conflict of interest.

\section{ACKNOWLEDGMENTS}

Research supported by the grant from the National Nature Science Foundation of China (\#80151459), the Development Project from Science and Technology Department of Jilin Province (\#140520020JH), and the Thirteen Five Science and Technology Research Project of Jilin Province Department of Education (\#2016-467).

\section{REFERENCES}

\footnotetext{
Abbruzzese C, Diodoro MG, Sperduti I, Mileo AM, et al. (2015). Detection of phosphorylated insulin receptor in colorectal adenoma and adenocarcinoma: implications for prognosis and clinical outcome. J. Cell. Physiol. 230: 562-567. http:// dx.doi.org/10.1002/jep. 24733
}

Genetics and Molecular Research 16 (1): gmr16019272 
Balog CI, Stavenhagen K, Fung WL, Koeleman CA, et al. (2012). N-glycosylation of colorectal cancer tissues: a liquid chromatography and mass spectrometry-based investigation. Mol. Cell. Proteomics 11: 571-585. http://dx.doi. org/10.1074/mcp.M111.011601

El-Karim EA, Hagos EG, Ghaleb AM, Yu B, et al. (2013). Krüppel-like factor 4 regulates genetic stability in mouse embryonic fibroblasts. Mol. Cancer 12: 89. http://dx.doi.org/10.1186/1476-4598-12-89

Fu JW and Chu XQ (2015). Correlation between non-metastatic protein 23 expression and clinicopathological features of colorectal cancer in Asians. Genet. Mol. Res. 14: 15597-15608. http://dx.doi.org/10.4238/2015.December.1.11

Ghaleb AM, McConnell BB, Nandan MO, Katz JP, et al. (2007). Haploinsufficiency of Krüppel-like factor 4 promotes adenomatous polyposis coli dependent intestinal tumorigenesis. Cancer Res. 67: 7147-7154. http://dx.doi. org/10.1158/0008-5472.CAN-07-1302

Jiang H, Fu XG and Chen YT (2015). Serum level of endothelial cell-specific molecule-1 and prognosis of colorectal cancer. Genet. Mol. Res. 14: 5519-5526. http://dx.doi.org/10.4238/2015.May.25.3

Kwon CH, Park HJ, Choi JH, Lee JR, et al. (2015). Snail and serpinA1 promote tumor progression and predict prognosis in colorectal cancer. Oncotarget 6: 20312-20326. http://dx.doi.org/10.18632/oncotarget.3964

Lee HY, Ahn JB, Rha SY, Chung HC, et al. (2014). High KLF4 level in normal tissue predicts poor survival in colorectal cancer patients. World J. Surg. Oncol. 12: 232. http://dx.doi.org/10.1186/1477-7819-12-232

Li D, Peng Z, Tang H, Wei P, et al. (2011). KLF4-mediated negative regulation of IFITM3 expression plays a critical role in colon cancer pathogenesis. Clin. Cancer Res. 17: 3558-3568. http://dx.doi.org/10.1158/1078-0432.CCR-10-2729

Li LN, Jiang KT, Tan P, Wang AH, et al. (2015a). Prognosis and clinicopathology of CXCR4 in colorectal cancer patients: a meta-analysis. Asian Pac. J. Cancer Prev. 16: 4077-4080. http://dx.doi.org/10.7314/APJCP.2015.16.9.4077

Li WX, Xiao HW, Hong XQ and Niu WX (2015b). Predictive value of CK20 in evaluating the efficacy of treatment and prognosis after surgery for colorectal cancer. Genet. Mol. Res. 14: 5823-5829. http://dx.doi.org/10.4238/2015.May.29.14

Liu C, La Rosa S and Hagos EG (2015). Oxidative DNA damage causes premature senescence in mouse embryonic fibroblasts deficient for Krüppel-like factor 4. Mol. Carcinog. 54: 889-899. http://dx.doi.org/10.1002/mc.22161

Livak KJ and Schmittgen TD (2001). Analysis of relative gene expression data using real-time quantitative PCR and the 2(-Delta Delta C(T)) Method. Methods 25: 402-408. http://dx.doi.org/10.1006/meth.2001.1262

McConnell BB and Yang VW (2010). Mammalian Krüppel-like factors in health and diseases. Physiol. Rev. 90: 13371381. http://dx.doi.org/10.1152/physrev.00058.2009

Patel NV, Ghaleb AM, Nandan MO and Yang VW (2010). Expression of the tumor suppressor Krüppel-like factor 4 as a prognostic predictor for colon cancer. Cancer Epidemiol. Biomarkers Prev. 19: 2631-2638. http://dx.doi. org/10.1158/1055-9965.EPI-10-0677

Schoenhals M, Kassambara A, Veyrune JL, Moreaux J, et al. (2013). Krüppel-like factor 4 blocks tumor cell proliferation and promotes drug resistance in multiple myeloma. Haematologica 98: 1442-1449. http://dx.doi.org/10.3324/ haematol.2012.066944

Shin SH, Kwon YW, Heo SC, Jeong GO, et al. (2014). Krüppel-like factor 4 mediates lysophosphatidic acid-stimulated migration and proliferation of PC3M prostate cancer cells. Exp. Mol. Med. 46: e104. http://dx.doi.org/10.1038/ emm.2014.34

Shinmura K, Kato H, Kawanishi Y, Nagura K, et al. (2015). SASS6 overexpression is associated with mitotic chromosomal abnormalities and a poor prognosis in patients with colorectal cancer. Oncol. Rep. 34: 727-738.

Song E, Ma X, Li H, Zhang P, et al. (2013). Attenuation of krüppel-like factor 4 facilitates carcinogenesis by inducing g1/s phase arrest in clear cell renal cell carcinoma. PLoS One 8: e67758.http://dx.doi.org/10.1371/journal.pone.0067758

Szczepanik AM, Siedlar M, Szura M, Kibil W, et al. (2015). Preoperative serum chemokine (C-C motif) ligand 2 levels and prognosis in colorectal cancer. Pol. Arch. Med. Wewn. 125: 443-451.

Ueda M, Iguchi T, Nambara S, Saito T, et al. (2015). Overexpression of transcription termination factor 1 is associated with a poor prognosis in patients with colorectal cancer. Ann. Surg. Oncol. 22 (Suppl 3): S1490-S1498. http://dx.doi. org/10.1245/s10434-015-4652-7

Weng W, Feng J, Qin H and Ma Y (2015). Molecular therapy of colorectal cancer: progress and future directions. Int. J. Cancer 136: 493-502.

Zhang G, Zhu H, Wang Y, Yang S, et al. (2009). Krüppel-like factor 4 represses transcription of the survivin gene in esophageal cancer cell lines. Biol. Chem. 390: 463-469. http://dx.doi.org/10.1515/BC.2009.060

Zhang N, Zhang J, Wang ZW, Zha L, et al. (2012). Altered expression of Krüppel-like factor 4 and $\beta$-catenin in human gastric cancer. Oncol. Lett. 3: 1017-1022.

Zhang Z, Wang M, Zhou L, Feng X, et al. (2015). Increased HMGB1 and cleaved caspase-3 stimulate the proliferation of tumor cells and are correlated with the poor prognosis in colorectal cancer. J. Exp. Clin. Cancer Res. 34: 51. http:// dx.doi.org/10.1186/s13046-015-0166-1

Zheng H, Pritchard DM, Yang X, Bennett E, et al. (2009). KLF4 gene expression is inhibited by the notch signaling pathway that controls goblet cell differentiation in mouse gastrointestinal tract. Am. J. Physiol. Gastrointest. Liver Physiol. 296: G490-G498. http://dx.doi.org/10.1152/ajpgi.90393.2008

Zhou Y, Hofstetter WL, He Y, Hu W, et al. (2010). KLF4 inhibition of lung cancer cell invasion by suppression of SPARC expression. Cancer Biol. Ther. 9: 507-513. http://dx.doi.org/10.4161/cbt.9.7.11106

Genetics and Molecular Research 16 (1): gmr16019272 